\title{
Development and Experimentation of an Articulated Mechanical System using Internet
}

\author{
F. Z. ZENDAOUI*, M.O. MAHMOUDI**, A. ZAATRI*** \\ *Nuclear Research Center of Birine, BP. 18017200 Ain-Oussera, Djelfa, Algeria. E-mail: zohrazend@hotmail.com \\ **Laboratory of Process Control, Polytechnical National School, Algeria, E-mail: momahmoudi@yahoo.fr \\ ***Advanced Technology Laboratory, Mentouri University of Constantine, Algeria, E-mail: azaatri@yahoo.com \\ cross $^{\text {ref }}$ http://dx.doi.org/10.5755/j01.mech.4.24.19386
}

\section{Introduction}

Historically, the development of tele robotics systems has been stimulated since the Second World War by the need for human intervention to perform tasks in hazardous and in hostile environments.

Teleoperation systems have been used for decades especially for tasks carried out in nuclear reactors when radiation and the risk of contamination often impede hands on work. Later on, many other applications have renewed the interest on this topic. We can note the space exploration [9], undersea operation, tele surgery, tele manufacturing and teleoperation processes in nuclear environment [10]. More recently, tele robotics systems have shifted to be a central technology under the pressure of unmanned systems such as drones, modern military vehicles, etc.

From the telecommunication point of view, most applications require special networks. It must be mentioned that time delays are a main cause of instability for tele robotics systems. However, nowadays, many tele robotics systems can use the most popular network Internet $[1,2]$. Nevertheless, it is well known that Internet presents a number of limitations such as restricted bandwidth and the random time delays between the operational site and the operator which render more difficult the teleoperations. In fact, other sources include noise, external disturbances, a lack of luminosity, shades, and even the operator experience [3, 4]. In fact, various encountered problems remain to be solved for internet-based tele robotics systems since they directly influence their performances. To solve these issues, some systems offer a combination of control mode or multimodality $[5,6]$. Other techniques use augmented reality, virtual reality and may add artificial intelligence functions to support operators $[7,8]$.
In this paper we present, a client-server tele robotics application which makes it possible for operators to remotely handle tasks with a remote serial robot.

The interaction between the operator and the working environment are accomplished via a graphical user interface allowing the control, the monitoring, the supervision and the management of the tasks. A lot of attention was paid to the design of the graphical user interface.

A presentation of the implementation of the robot control functions will be presented enabling tasks such as pick-and-place type. Some modern and interactive control modes are implemented namely control modes Mouseclick-based control [11], Image-Based Control [12, 13] and Gestural-based control [14, 15].

Our tele robotics system has been tested for various tasks performed in local site as well as in remote sites with distances of hundreds of kilometres. Encouraging results have been obtained, enabling our system to be adapted for a wider use in the manufacturing sector and for nuclear applications

\section{Design and implementation of the tele robotics system}

The design concerns a manipulator arm which is constituted of 3 dofs with a kinematics structure of RRR type holding a gripper in his end-effector. On the other hand, we have designed a pan-tilt unit (PTU) to control a webcam for selecting views. Thus, a Graphical User Interface has been designed with the intention of making it easy for operators to interact with the work environment.

Schematic illustration of the system is shown in Fig. 1.

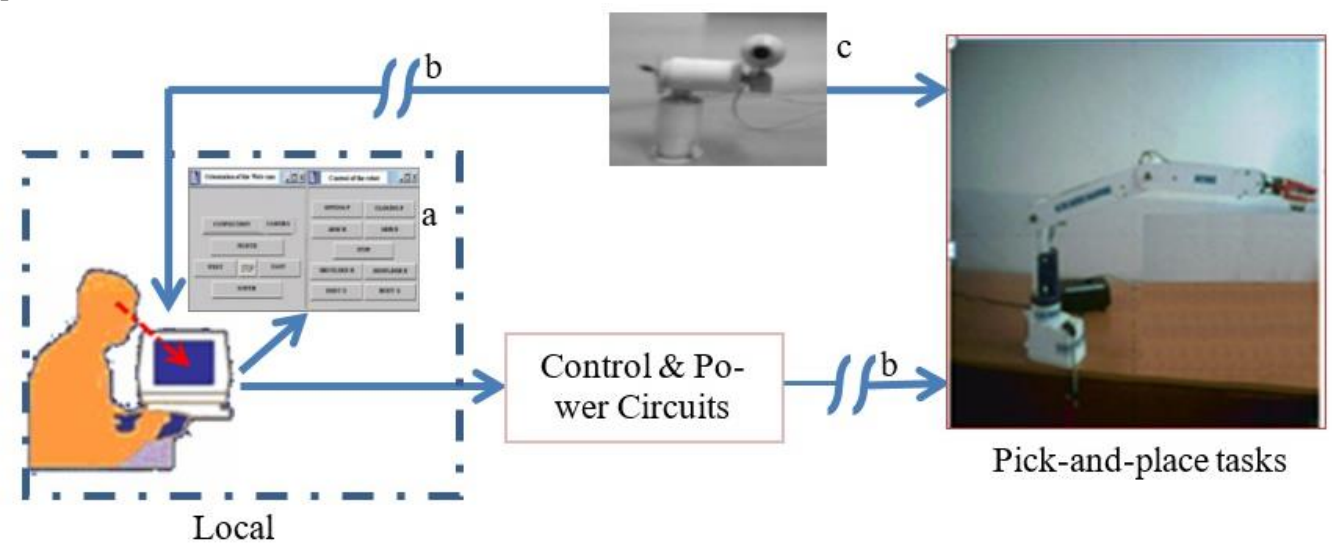

Fig. 1 The tele robotic remote system: a - Graphical User Interface, b - Network, c - Camera Pan-tilt 


\subsection{Operational system description}

The local site includes a human operator using a PC. In the operational site, we find the manipulator arm, the video camera, thus the communication module with the server; this last contains the control programs of the robot and the routines of capture and image processing.

The vision system, based in a pan-tilt camera, allows the transmission of images of the work environment (Robot). The human operator, based on these received images, directs remotely the tasks through a Graphical User Interface.

We centred our work for the pick-and-place operations; in this case we have simply implemented the direct and inverse geometrical model that has proved sufficient for remote handling operations for our applications.

\subsection{Human-robot interface}

The control and supervision of the robot is done through the control panel interface (Fig. 2). A simple interface is building up to provide as much information as possible for teleoperation. This user interface consists of several Java Applets; it achieves several functionalities. In addition to ensuring the connection and the disconnection of the client to the server, it allows separately the order of different elements of the manipulator robot: the body, the shoulder, the arm of the manipulator robot and opening or closing the gripper "end effector". It allows also the ordering and the orientation of the camera in the four directions (East, West, North and South).

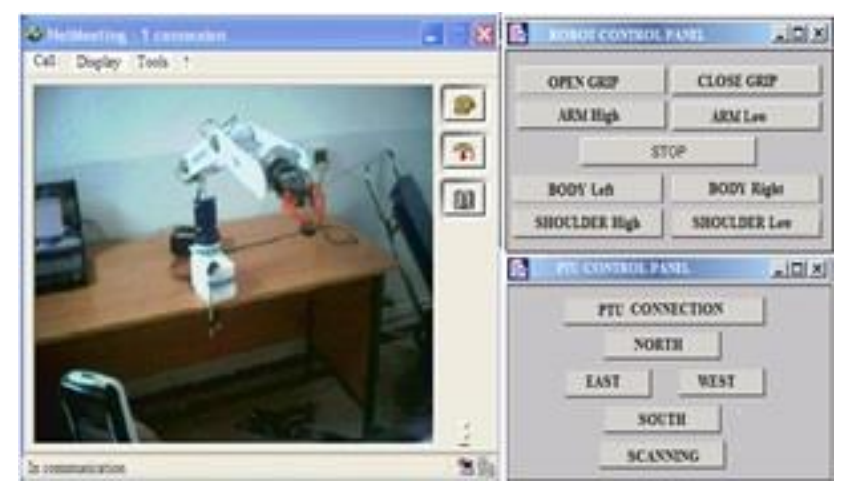

Fig. 2 Control panels (Robot an ptu)

The visual information feedback is carried out using the images transmitted by the camera by means of NetMeeting. This makes it possible to the operator to locate the grip compared to the objects to be handled.

By means of this graphical user interface, a simulation program was conducted to test the implementation of the robot control manipulator by using the inverse and the direct geometric models, which proves quite sufficient to pick-and-place applications.

\section{Modelling and simulation}

Modelling robots systematically and automatically requires an adequate method for the description of their morphology. Several methods and notations have been proposed [16]. The most common is that of Denavit-Hartenberg [17], developed for simple open structures.
The execution of a task in robotics requires to position and to direct the end-effector, according to the instructions of the operator. This one must specify the operational or the articular co-ordinates of the gripper.

To this end, the direct and the inverse geometrical models upon which the robot control will be based are presented hereafter (Fig. 3).

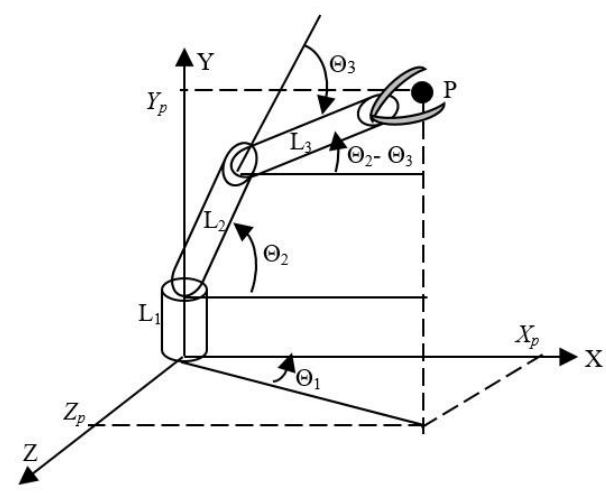

Fig. 3 Control panels (Robot an ptu)

The robot control programs, as well as the robot simulation, are written in java language. The operator performs the task by means of a graphical panel by clicking on the corresponding button (Fig. 2).

\subsection{Direct geometrical model}

The design and control of robots require the calculation of some mathematical models, such as: - The model transformation between the operational space (in which is defined the position of the end effector) and the joint space (in which is defined a robot configuration). We distinguish:

1. The forward and reverse geometric models who express the position of the end effector allowing to the joint variables of the mechanism and vice versa;

2. The forward and reverse kinematic models that express the velocity of the end effector according to the joint and vice versa speeds;

3. Dynamic models defining the equations of motion of the robot, which establish the relationship between the torques, and forces exerted by the actuators and the positions, speeds and accelerations of the joints. In our application, we have implemented a control based on the geometric model, which has proven sufficient for remote handling operations. The direct geometrical model links the articular co-ordinates to the spatial co-ordinates. In a general form, it can be expressed as follows:

$$
X=f(\theta)
$$

where: $X=\left(X_{p}, Y_{p}, Z_{p}\right)^{t}$, are operational co-ordinates vector of the end-effector. And $\theta=\left(\theta_{1}^{R}, \theta_{2}^{R}, \theta_{3}^{R}\right)^{t}$, are vector represents the articular co-ordinates.

Since the robot is of type $R R R$ (three rotations), then we consider a reference frame $R(X, Y, Z)$ situated at the robot base. We associate a frame $R i$ to each segment. Thus the operational co-ordinates of the end-effectors are given by these equations: 


$$
\left[\begin{array}{l}
X_{p}=\left(L_{2}^{R} \cos \theta_{2}^{R}+L_{3}^{R} \cos \left(\theta_{2}^{R}+\theta_{3}^{R}\right)\right) \cos \theta_{1}^{R} \\
Y_{p}=L_{2}^{R} \sin \theta_{2}^{R}+L_{2}^{R} \sin \left(\theta_{2}^{R}+\theta\right)_{3}^{R}+L_{1}^{R} \\
Z_{p}=\left(L_{2}^{R} \cos \theta_{2}^{R}+L_{3}^{R} \cos \left(\theta_{2}^{R}+\theta_{3}^{R}\right)\right) \sin \theta_{1}^{R}
\end{array}\right],
$$

where: $L_{1}^{R}, L_{2}^{R}$ and $L_{3}^{R}$ are the lengths of the links.

Similarly, since the camera has two dofs of type $R R$ (Fig. 4), we obtain the relationships between the operational co-ordinates and the articular co-ordinates as given below.

$$
\left[\begin{array}{l}
X_{c}=\left(L_{2}^{c} \cos \theta_{1}^{c}\right) \cos \theta_{2}^{c} \\
Y_{c}=\left(L_{2}^{c} \sin \theta_{1}^{c}\right) \cos \theta_{2}^{c} \\
Z_{c}=L_{1}^{c}+L_{2}^{c} \sin \theta_{2}^{c}
\end{array}\right] .
$$

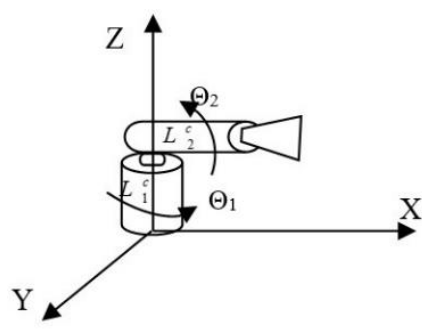

Fig. 4 The Pan-Tilt Unit coordinates

\subsection{Inverse geometrical model}

The resolution of the inverse kinematic problem of a manipulator is paramount for control in Cartesian space. The inverse kinematics is to determine the coordinates of the joint actuators to achieve a position and orientation of the effector in cartesian space Eq.(4).

$$
\left[\begin{array}{l}
\theta_{1}^{R}=\operatorname{artg} \frac{Z_{p}}{X_{p}} \\
\theta_{2}^{R}=\operatorname{arcos} \frac{\left[\frac{X_{p}}{\cos \theta_{1}^{R}}\left(L_{2}+L_{3} \cos \theta_{3}^{R}\right)\right]-\left[\left(Y_{p}-L_{1}\right)\left(L_{3} \sin \theta_{3}^{R}\right)\right]}{L_{2}^{2}+L_{3}^{2}+2 L_{2} L_{3} \cos \theta_{3}^{R}} \\
\theta_{3}^{R}=\operatorname{arcos} \frac{\left[\frac{X_{p}}{\cos \theta_{1}^{R}}\right]^{2}+\left(Y_{p}-L_{1}\right)^{2}-L_{2}-L_{3}}{2 L_{2} L_{3}}
\end{array}\right]
$$
the camera is

Similarly, the inverse geometrical coordinates for

$$
\left[\begin{array}{l}
\theta_{1}^{c}=\operatorname{artg} \frac{Y_{c}}{X_{c}} \\
\theta_{2}^{c}=\operatorname{arsin}\left(\frac{Z_{c}-L_{1}^{c}}{L_{2}^{c}}\right)
\end{array}\right] .
$$

These equations of the direct and inverse geometric models have been implemented for simulation and to control the robot. The subscript "R" refers to the robot and "C" of the camera.

\subsection{Simulation in $2 \mathrm{D}$ space}

The simulation program enables to pre-verify the accomplishment of the commands (Fig. 5).

The commands can be given as or operational coordinates (inverse and direct geometrical models).

\section{Experiments of the manipulator arm}

We implemented the model of equations, previously defined, to control the robot. The operator performs the task by means of a graphical panel by clicking on the corresponding button. On the other hand, the operator can direct the webcam by remotely controlling the pan-tilt unit in different directions: North, South, East and West.

The experiments carried out relate two different situations: with direct vision of the site of work by the operator, or without direct vision by internet interconnection (Fig. 6).

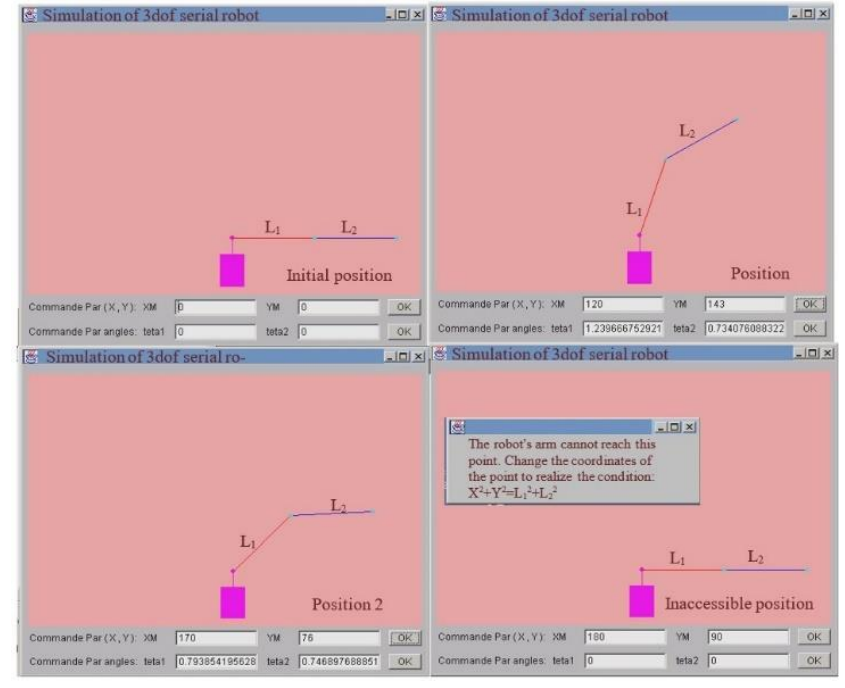

Fig. 5 Command of the robot position using articular co-ordinates

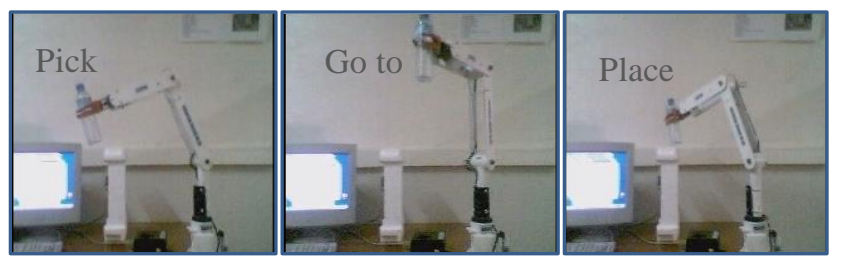

Fig. 6 Realisation of the pick-and-place task by the manipulator arm

The control modes carried out with our system are developed below. 


\section{1. Mouse-click-based control}

With this mode, we can control the robot and the camera by simple mouse clicks on the appropriate buttons of a panel. Each button represents a specific function or a specific direction of motion (Fig. 2).

Within this control mode, the operator can carry out pick-and-place tasks such as pick a bottle from above a table and place it at another location (Fig. 6). To achieve tasks with this mode, the operator directs the robot by a series of clicks on the appropriate buttons.

\subsection{Image-based control}

This mode of control enables high level control. In this mode, the operator directs the robot towards locations in $2 \mathrm{D}$ or $3 \mathrm{D}$ space by moving only their images using clicks of a mouse.

From the received images, the operator specifies the target object with a simple click, the vision system considers these coordinates and the robot can move towards the designated object in the image, a second click on the control panel webcam directs it to where we want to release the object, the image received by the camera we point the place showing the positioning of the object. This technique is both powerful and necessary to lift many difficulties and disadvantages associated with the tele operator.

Some experiments have been carried out using the image-based control. Practically, this control mode is used to send the robot to some location. First, an image of the remote site is grabbed. Then, the operator selects an object of interest. The stereovision software extracts the coordinates of this object which are used to move the robot towards the object in the real world.

\subsection{Gestural-based control}

In this mode of command, the gestural movement is presented as a means to control a robot from a sequence of images transmitted by a camera. The experimental system consists of a computer, a webcam camera and a robot with three degrees of freedom.

The algorithm developed for the pursuit of moving objects use the KLT tracker for the determination of matching points in the image sequences, to orient the robot in the corresponding direction.

Experiments have been carried out within this control mode. The operator generates a series of movements in different directions. The software analyses the image stream and moves the robot in the corresponding directions.

This control mode offers the advantage of being without contact of the operator with the computer. Another advantage is the possibility of using this technique for robot programming by human demonstration. Nevertheless, some difficulties which are related to image processing and environment issues limit the capability of this control mode.

\section{Conclusion and perspectives}

This paper has described the design, the implementation of our tele robotics system and the results of its use for performing some preliminary remote pick-and-place tasks.

An important added value is the use of the modern interactive control modes (mouse-click-based, image-based, and gestural-based). The different modes have been integrated into a multimodal interface that has been built up according to the user-centred design enabling to use them individually or in a sequential combination. This capability enhances the system flexibility with respect to the operator and enables to perform various complex tasks in different environments.

Tested in local sites and in remote sites over hundreds of kilometers through Internet, the obtained results are encouraging and confirm the effective possibility to use this tele robotics system for carrying out tasks for various industrial applications such as nuclear, welding operations, intervention and exploration in hostile environment.

The system presents a modular architecture that enables its extension to other control modes such as speech control, myoelectric control, mind control, etc.

However, some problems had been encountered during our experiments that affect the system performance. One can notice the transmission delays which cause control difficulties and instabilities. Other issues are related to the human operator which is due to the visual feedback which can be resolved by adding more sensors.

As perspectives, some improvements are actually under developments that can support the operator. In this context, one can note a more flexible coordination between modes; the integration of virtual reality, augmented reality and some techniques of artificial intelligence. Moreover, some aspects of human factors have to be considered for ensuring more adaptability, safety, etc.

\section{References}

1. Stein, M. 2000, Interactive internet artistry, painting on the World Wide Web with the Puma Paint project, IEEE Robotics and Automation Magazine 7(1): 28- 32. https://doi.org/10.1109/100.848265.

2. Cui; J.; Tosunoglu; S.; Roberts, R.; Moore, C.; Repperger, D. W. 2003. A Review of Teleoperation System, Proceedings of the Florida Conference on Recent Advances in Robotics, FCRAR, Boca Raton, Florida.

3. Kamrani, E.; Momeni, H. R.; Sharafat, A. R. 2005. A Novel Adaptive Control System for Stable Teleoperation via Internet, Proceedings of IEEE Conference on Control Applications, Toronto.

4. Kamrani Ramazani, A.; Monteiro, F. 2006. Teleoperation via Internet with Time-Varying Delay, Proceedings of the 13th IEEE International Conference on Electronics, Circuits and Systems (ICECS), NICE: 736-739.

5. Zaatri, A.; Oussalah, M. 2003. Integration and design of multimodal interfaces for supervisory control systems, Information fusion journal E: 135-150.

6. Kaber, D B.; Wright, MC.; Sheik-Nainar, M.A. 2006. Investigation of multi-modal interface features for adaptive automation of a human-robot system, International Journal of Human-Computer Studies 64(6): 527-540. https://doi.org/10.1016/j.ijhcs.2005.11.003.

7. Leutert, F.; Schilling, K. 2012. Support of Power Plant Telemaintenance with Robots by Augmented Reality Methods, 2nd International Conference on Applied Robotics for the Power Industry (CARPI) ETH Zurich, Switzerland.

8. Zhijiang, D.; Zhiheng, J.; Minxiu, K. 2005. Virtual Reality-based Telesurgery via Teleprogramming 
Scheme Combined with Semi-autonomous Control, Engineering in Medicine and Biology Society, IEEEEMBS: 2153-2156.

9. Eastlund, B.J.; Jenkins, L.M. 2004. Mission for Planet Earth: defining a vision for the space program, Aerospace Conference, Proceedings IEEE 1: 6-13.

10. Bakari, M. J.; Zied, K. M.; Seward, D. W. 2007. Development of a Multi-Arm Mobile Robot for Nuclear Decommissioning Tasks, International Journal of Advanced Robotic Systems 4(4): 387-406. https://doi.org/10.5772/5665.

11. Zaatri, A. A Low Cost Experimental Telerobotic Systems, Mechanical Department Laboratory, University Mentouri of Constantine, Algeria.

12. Abdelkader, H.; Mezouar, H.; Andreff, Y.; Martinet, P. 2005. Image-based control of mobile Robot, with Central Catadioptric Cameras, in: Proceedings of the IEEE International Conference on Robotics and Automation, ICRA'05, Barcelona, Spain, 3533-3538. https://doi.org/10.1109/ROBOT.2005.1570655.

13. Ni, T.; Yamada, H.; Zhang, H. 2010. Image based real-time 3D reconstruction for teleoperation system, IEEE, Computer, Mechatronics, Control and Electronic Engineering (CMCE) 2: 265-268.

14. Frigola, M.; Rodriguez, A.; Amat, J.; Casals, A. 2007. Computer Vision Body Modelling for Gesture Based Teleoperation, Advances in Telerobotics, STAR 31, Springer-Verlag Berlin Heidelberg, 121-137.

15. Ondrousek, Vit. 2016. Control of the End Effector Position Based on Motion Capture, Springer International Publishing Switzerland. https://doi.org/10.1007/978-3-319-23923-1_80.

16. Khalil, W.; Dombre, E. 2002. Modelisation, identification and control of robots, Hermes Penton Science. London, ISBN 1-90399-613-9, 480.

17. Hartenberg, R.S. 1955. A kinematic notation for lower pair mechanism based on matrices, Trans. of ASME, J. of Applied Mechanics 22: 215-221.
F. Z. Zendaoui, M.O. Mahmoudi, A. Zaatri

\section{DEVELOPMENT AND EXPERIMENTATION OF AN ARTICULATED MECHANICAL SYSTEM USING THE INTERNET}

S u m m a r y

The objective of our paper is to design, implement and test an experimental telerobotics system dedicated to perform pick-and-place tasks in local as well as in remote sites via Internet connection.

The interaction between the operator site and the remote site is ensured via a graphical user interface which integrated some control modes and enables interaction, monitoring and supervision of task accomplishment.

To allow flexibility with respect to the operator as well as to the task performance, the user interface has been designed according to the principles of user-centred design. It includes three modern interactive control modes namely mouse-click-based, image-based, gestural-based controls.

Experimental tasks have been carried out to test our tele robotics system. The obtained successful results even at large distances are very despite the complexity of the tasks and of the tele robotics system.

Key words: Tele robotics; Internet-based; Mouse-clickbased control; Image-based control; Gestural-based control.

Received October 29, 2017

Accepted August 20, 2018 